\title{
Detection of SARS-CoV-2 antibodies in pediatric patients: An Iranian referral hospital-based study
}

\author{
Setareh Mamishi ${ }^{\mathrm{a}, \mathrm{b}}$, Golnaz Ghazizadeh Esslami ${ }^{\mathrm{c}, \mathrm{d}, \mathrm{e}}$, Mohsen Mohammadi ${ }^{\mathrm{f}}$, \\ Mohammad Reza Abdolsalehi ${ }^{\mathrm{a}}$, Reihaneh Hosseinpour Sadeghi ${ }^{\mathrm{b}}$, Shima Mahmoudi ${ }^{\mathrm{b}, \mathrm{g}, *}$ and \\ Babak Pourakbari ${ }^{\mathrm{b}, \mathrm{g}, *}$ \\ ${ }^{a}$ Department of Infectious Diseases, Pediatrics Center of Excellence, Children's Medical Center, Tehran University \\ of Medical Sciences, Tehran, Iran \\ ${ }^{\mathrm{b}}$ Pediatric Infectious Disease Research Center, Tehran University of Medical Sciences, Tehran, Iran \\ ${ }^{\mathrm{c}}$ Department of Emergency, Pediatrics Center of Excellence, Children's Medical Center, Tehran University of \\ Medical Sciences, Tehran, Iran \\ ${ }^{\mathrm{d}}$ Department of Newborn Nursery, Neonates, and Pediatrics, Ziaeian Hospital, Tehran University of Medical \\ Sciences, Tehran, Iran \\ ${ }^{\mathrm{e}}$ Department of Family Medicine, Ziaeian Hospital, Tehran University of Medical Sciences, Tehran, Iran \\ ${ }^{\mathrm{f}}$ Non-Communicable Pediatric Diseases Research Center, Health Research Institute, Babol University of Medical \\ Sciences, Babol, Iran \\ 'Pediatrics Center of Excellence, Children's Medical Center, Tehran University of Medical Sciences, Tehran, Iran
}

\begin{abstract}
.
BACKGROUND: As the extent of the pandemic and its seroprevalence pattern has been less clarified in pediatrics to date, we aimed to conduct this study to investigate the clinical and laboratory characteristics of COVID-19 in Iranian children, with a focus on evaluating the antibody prevalence and its relation with the laboratory tests.

METHODS: All children with highly suspected COVID-19 were included. Anti-nucleoprotein SARS-CoV-2 were measured using SARS-CoV-2 immunoglobulin M (IgM) and SARS-CoV-2 IgG ELISA kits. Hypothesis testing was carried out through SPSS to unravel any association between the measurement tools and important clinical and laboratory characteristics.

RESULTS: In this study, 254 patients were evaluated and 117 cases (46\%) were male. The nucleic acid detection results for patient 55 were negative, but the IgM and IgG results were positive. Totally, 190 patients were tested for IgM in which only $14(7.3 \%)$ had positive tests. Positive IgG was detected in 51 (20\%) out of 254 patients; among them, 30 patients had negative SARS-CoV-2 RT-PCR (59\%). Lower level of platelets in IgG positive group in comparison with the IgG negative group was observed (P value: 0.015). Moreover, higher alanine aminotransferase (ALT) was observed in the in IgG positive group ( $P$ value: 0.02). In patients with positive IgM, relative hypocalcemia (median of 8.25; IQR: 8.02-8.62) was found which appeared to be significant ( $P$ value: 0.02 ).

CONCLUSION: This is the first largest study describing the SARS-CoV-2 seropositivity among children in Iran and provides important insight about the COVID-19 infection in children.
\end{abstract}

Keywords: SARS-CoV-2, COVID-19, children, antibody, IgM, IgG

\footnotetext{
*Corresponding authors: Shima Mahmoudi and Babak Pourakbari, Pediatric Infectious Disease Research Center, Pediatrics Center of Excellence, Children's Medical Center Hospital, Dr. Gharib Street,
}

Keshavarz Boulevard, Tehran, Iran. Tel.: +98 216642 8996; Fax: +98 216642 8996; E-mail: sh-mahmoudi@sina.tums.ac.ir (Shima Mahmoudi), pourakbari@sina.tums.ac.ir (Babak Pourakbari). 


\section{Introduction}

The novel coronavirus (COVID-19) outbreak has evolved into a novel public health disaster, which the World Health Organization (WHO) has classified as a pandemic since March 11, 2020 [1]. However, the actual infection rates are probably similar to those of adults, even if the actual prevalence in children is difficult to quantify due to different symptoms, lack of consideration or less severe course of the disease [2,3]. Mentioned facts have led to fewer laboratory assessments in children and fewer documented cases consequently. Nevertheless, the clinical course of the disease caused by severe acute respiratory syndrome coronavirus 2 (SARS-CoV-2), particularly the pulmonary involvement, appears less severe than in adults, which could be consistent with both host factors and exposure. Furthermore, death in this age group is rare but it can occur due to life-threatening conditions such as multisystem inflammatory syndrome in children (MISC) [4-6].

The rapid development of diagnostic and preventive protocols is inspected as one of the precious achievements of the COVID-19 pandemic. For suspected patients, real-time polymerase-chain-reaction (RT-PCR) should be performed to detect the positive nucleic acid of SARS-CoV-2 in sputum, throat swabs, and secretions of the lower respiratory tract samples [7]. However, due to the low sensitivity and specificity of molecular testing, other laboratory parameters and serological tests, as well as CT imaging of the lungs, are required if molecular test results do not match clinical manifestation [8].

It is claimed that unlike adults having anti-spike (S) IgG, IgM and IgA antibodies, as well as antinucleocapsid (N) IgG antibody [9], children with and without MIS-C predominantly generate IgG antibodies specific for the $\mathrm{S}$ protein but not the $\mathrm{N}$ protein. Additionally, children with and without MIS-C had decreased neutralizing activity in comparison with adults, suggestive of a reduced protective serological response [10]. On the other hand, some studies are indicative of similar antibody titers in response to SARSCoV-2, irrespective of age [11].

As, the extent of the pandemic and its seroprevalence pattern has been less clarified in pediatrics to date [11], we aimed to conduct this study to investigate the clinical and laboratory characteristics of COVID-19 in Iranian children, with a focus on quantifying the antibody prevalence and its connection with the disease features.

\section{Materials and methods}

\subsection{Study design}

This research was approved by the Ethics Committee of Tehran University of Medical Sciences, Tehran, Iran (IR.TUMS.VCR.REC.1399.057).

\subsection{Study population}

All symptomatic children with suspected COVID-19 who were admitted to Children's Medical Centre hospital, the most experienced and sub specialized hospital considered as a referral center for pediatric COVID-19 cases in Iran, were included between February 2020 and January 2021.

Suspected cases of COVID-19 were defined according to the presence of at least one of the following criteria: (1) a history of contact with people with COVID-19 (positive SARS-CoV-2 nucleic acid test) within 14 days before onset; (3) a history of contact with patients infected with COVID-19 or a history of contact with people with fever or respiratory symptoms.

Symptomatic children were defined as those with fever and/or respiratory signs such as cough, dysphagia, rhinorrhea, diarrhea, vomiting, cutaneous signs, taste loss and/or anosmia during the last 7 days. They were only subjected to blood sampling if informed consent was obtained and signed by their parents/legal guardians, regardless of the study.

We considered MIS-C diagnosis according to the following criteria: 1) history of close SARS-CoV-2 contact, 2) the presence of fever $\left(>38^{\circ} \mathrm{C}\right)$ lasting for more than $24 \mathrm{~h}, 3$ ) signs/symptoms of at least 2 organ involvement, 4) laboratory results displaying systemic inflammation [12].

\subsection{Measurements}

A Reverse transcription polymerase chain reaction (RT-PCR) test for SARS-CoV-2 was performed using a nasopharyngeal swab for all of the patients [13,14]. The RNA of the collected samples on the swab was then extracted using a SinaPure Viral kit (Sinaclon, Iran) and cDNA template synthesis was accomplished using PrimeScript RT reagent kit (taKaRa, Japan). The reverse transcription polymerase chain reaction (RTPCR) was performed according to the CDC protocol. The PCR cycle was run as follows: $95^{\circ} \mathrm{C}$ for $3 \mathrm{~min}$, followed by 45 cycles of $95^{\circ} \mathrm{C}$ for $3 \mathrm{~s}$, and $58^{\circ} \mathrm{C}$ for 
Table 1

SARS-CoV-2 RT-PCR and serological assays

\begin{tabular}{|c|c|c|c|c|c|}
\hline & \multicolumn{2}{|c|}{ Patients non-MIS-C $(N=215)$} & \multicolumn{2}{|c|}{ Patients with MIS-C $(N=39)$} & \multirow[t]{2}{*}{$P$ value } \\
\hline & $N$ & $\%$ & $N$ & $\%$ & \\
\hline SARS-CoV-2 RT-PCR & 80 & 37 & 10 & 26 & 0.2 \\
\hline Anti-N SARS-CoV-2 IgM & 12 out of 164 & 7 & 2 out of 26 & 7 & 1 \\
\hline Anti-N SARS-CoV-2 IgG & 43 & 20 & 8 & 20.5 & 1 \\
\hline Anti-N SARS-CoV-2 IgM and/or anti-N IgG & 50 & 23 & 10 & 26 & 0.83 \\
\hline
\end{tabular}

30 s. A cycle threshold value (Ct value) of less than 37 was defined as a positive test result.

After gaining the institutional review board approval, we acquired the residual blood samples left after essential samplings from these children and measured anti-N SARS-CoV-2 antibodies by using SARS-CoV-2 immunoglobulin M (IgM) ELISA kits (Pishtaz Teb, Iran, http://pishtazteb.com) and SARS-CoV-2 IgG ELISA kits (Pishtaz Teb, Iran http://pishtazteb.com) according to the manufacturer's protocol [14]. The results were reported as numeric values in the form of a ratio (the optical density of the sample/internal calibrator). A ratio of $\geqslant 1.1$ is considered positive (borderline from 0.8 and 1.09).

The demographic characteristics of patients, i.e., age, sex, comorbidities, known previous contacts with SARS-CoV-2 RT-PCR positive person, the history of possible COVID-19 symptoms and physical signs, time gap between fever onset and laboratory tests, laboratory findings (including leukocyte, neutrophil and lymphocyte counts, platelet count, C-Reactive Protein (CRP), troponin levels, Erythrocyte Sedimentation Rate (ESR), D-dimer, blood urea nitrogen (BUN), serum creatinine, liver enzymes, fibrinogen, ferritin, Lactate Dehydrogenase (LDH), and creatine phosphokinase (CPK)) were collected.

\subsection{Statistical analysis}

Statistical analyses were implemented using SPSS (Statistical Package for the Social Sciences) version 13.0 software (SPSS Inc.). All categorical variables were explained as frequency rates and percentages. Normally and non-normally distributed data is presented as mean \pm SD and medians (25th percentile and 75th percentile). Non-parametric tests and two-sided $\chi^{2}$-tests were used to compare the differences between groups, and a $P$-value $<0.05$ was considered statistically significant.

\subsection{Results}

In this study, 254 symptomatic patients with sus- pected COVID-19 were referred to Children's Medical Center between February 2020 and January 2021. Among them, 117 (46\%) were male. The age range was from 18 days to 16 years with a median of 5 years (IQR $2-10$ years). Forty percent of the patients $(n=101)$ had a history of contact with a person with confirmed COVID-19 in their family; half of the patients $(n=$ 128) had an underlying disease. The most common predisposing risk factors were cardiovascular diseases (30, $12 \%)$ and immunodeficiency disorders or malignancies $(35,14 \%)$. Fever $(n=215,85 \%)$, cough $(n=119$, $47 \%$ ), tachypnea ( $n=108,42.5 \%)$, and vomiting ( $n=$ $104,41 \%$ ) were listed as the most common clinical manifestations.

Lymphocytopenia (median $=1500$, IQR: 940-2800), $\mathrm{CRP}($ median $=21, \mathrm{IQR}: 5-49), \mathrm{ESR}($ median $=26$, IQR: 12-44), D-dimer (median = 0.7, IQR: 0.1-1.8) were the most common abnormal laboratory findings among our patients.

Severity of the disease was observed in 69 (27\%) of our patients. Moreover, ventilation assistance was required in $28(11 \%)$ of children and intubation was implemented in $16(6 \%)$ patients. ICU admission was required in 54 patients $(21 \%)$. Eventually, mortality was reported in $19(7 \%)$ of our study cases.

Out of 254 children with suspected COVID-19 disease, 90 (35\%) showed positive SARS-CoV-2 RT-PCR test and 39 patients $(15 \%)$ presented with MIS-c. The age of the SARS-CoV-2 RT-PCR positive and negative group was 8 years (IQR 5-15) and 5 years (IQR 4-8), respectively $(P=0.91)$. The nucleic acid detection results for patient 55 were negative, but the $\operatorname{IgM}$ and IgG results were positive. Totally, 190 patients were tested for IgM in which only 14 (7\%) had positive tests. Surprisingly, half of the patient with positive $\operatorname{IgM}(n=$ 7) had negative SARS-CoV-2 RT-PCR. Positive IgG was detected in 51 (20\%) out of 254 patients; among them, 30 patients had negative SARS-CoV-2 RT-PCR (59\%). Among 39 patients (15\%) presented with MIS-c, only $8(20.5 \%)$ had a detectable IgG antibody. Twentynine children (74\%) presenting with MIS-C display no detectable viral infection by SARS-CoV-2 RT-PCR (Table 1). The number of cases with anti-N IgG were similar in children with and without MIS-C. 
Table 2

Clinical finding of children with suspected COVID-19

\begin{tabular}{lccc}
\hline Symptom & $N(\%)$ in sero-negative group & $N(\%)$ in sero-positive group & $P$ value \\
\hline Fever & $160(82.5)$ & $55(92)$ & 0.10 \\
Cough & $88(46)$ & $31(52)$ & 0.46 \\
Sore throat & $11(6)$ & $8(13)$ & 0.09 \\
Conjunctivitis & $20(10)$ & $8(14)$ & 0.48 \\
Tachypnea & $86(44)$ & $22(37)$ & 0.37 \\
Chest pain & $10(5)$ & $3(5)$ & 0.63 \\
Rhinorrhea & $6(3)$ & $2(3)$ & 0.60 \\
Vomiting & $73(38)$ & $31(52)$ & 0.07 \\
Headache & $13(16)$ & $15(25)$ & 0.13 \\
Abdominal pain & $40(67)$ & $20(33)$ & 0.18 \\
Diarrhea & $51(26)$ & $20(33)$ & 0.321 \\
Respiratory distress & $87(45)$ & $22(37)$ & 0.30 \\
Myalgia & $6(77)$ & $14(23)$ & 0.86 \\
\hline
\end{tabular}

Table 3

Laboratory finding of the patients with suspected COVID-19 with different serology tests

\begin{tabular}{|c|c|c|c|c|c|c|}
\hline & IgG positive group & IgG negative group & $P$ value & IgM positive group & IgM negative group & $P$ value \\
\hline $\begin{array}{l}\text { White blood cell } \\
\text { count }\left(\times 10^{9} \text { cells }\right. \\
\text { per } \mathrm{L})\end{array}$ & $7.59(4.9-10.5)$ & $7.69(5.32-11.25)$ & 0.76 & $6.86(2.92-11.3)$ & $7.87(5.6-11.9)$ & 0.54 \\
\hline $\begin{array}{c}\text { Neutrophil count }(\times \\
\left.10^{9} \text { cells per } \mathrm{L}\right)\end{array}$ & $5.4(3.48-7.35)$ & $4.6(2.57-8.14)$ & 0.49 & $3.4(1.73-8.6)$ & $1.6(1.11-3.03)$ & 0.43 \\
\hline $\begin{array}{l}\text { Lymphocyte count } \\
\qquad\left(\times 10^{9} \text { cells per } \mathrm{L}\right)\end{array}$ & $1.4(0.92-2.92)$ & $1.6(0.98-2.82)$ & 0.39 & $0.79(0.6-2.76)$ & $5.2(2.85-8.18)$ & 0.13 \\
\hline $\begin{array}{l}\text { Platelet count }\left(\times 10^{9}\right. \\
\quad \text { cells per } \mathrm{L})\end{array}$ & $220.5(133-315.75)$ & $268(201-351.75)$ & 0.15 & $216(141.5-339)$ & $263(181.25-354.75)$ & 0.42 \\
\hline $\begin{array}{l}\text { Erythrocyte } \\
\text { sedimentation rate } \\
(\mathrm{mm} / \mathrm{h})\end{array}$ & $36.5(16.5-59.25)$ & $24(11-42.5)$ & 0.22 & $31(14.5-60.5)$ & $26(12-44.75)$ & 0.55 \\
\hline $\begin{array}{l}\text { C-reactive protein } \\
(\mathrm{mg} / \mathrm{L})\end{array}$ & $32(6.5-64.5)$ & $19(4-44.5)$ & 0.10 & $43(2-125)$ & $21(5-48)$ & 0.50 \\
\hline $\begin{array}{l}\text { Alanine } \\
\text { aminotransferase } \\
(\mathrm{U} / \mathrm{L})\end{array}$ & $23(17-39)$ & $18.5(12-41)$ & 0.02 & $17(14-39)$ & $21(13-44)$ & 0.64 \\
\hline $\begin{array}{l}\text { Aspartate } \\
\text { aminotransferase } \\
(\mathrm{U} / \mathrm{L})\end{array}$ & $32(26-38)$ & $30.5(21-42)$ & 0.20 & $34(25-42)$ & $30(23-40)$ & 0.40 \\
\hline $\begin{array}{l}\text { Lactate } \\
\text { dehydrogenase } \\
\text { (U/L) }\end{array}$ & 585.5 (459-699.75) & $544(416-727)$ & 0.16 & $690(539-957)$ & $544(430-707)$ & 0.21 \\
\hline $\begin{array}{l}\text { Creatine } \\
\text { phosphokinase } \\
\text { (U/L) }\end{array}$ & $55(31-101)$ & $66(39.5-107)$ & 0.55 & $55.5(34.5-96.25)$ & $62(38-98)$ & 0.48 \\
\hline Ferritin & 261.5 (118.75-549) & $144.5(77.5-553.5)$ & 0.34 & 337 (102.5-1440) & $149(76-577)$ & 0.57 \\
\hline Fibrinogen & $181(160.5-427)$ & $294(163.75-472.5)$ & 0.57 & $170(170-170)$ & $111175(0-323)$ & 1 \\
\hline Interleukin-6 & $3.3(1.5-43)$ & $1.3(0-15.4)$ & 0.54 & $15(15-15)$ & $3.2(0-15)$ & 0.48 \\
\hline
\end{tabular}

The median fever onset time before laboratory testing was 7 days (IQR 3.5-12) in children with IgM positive results, but 3 days (IQR 2-5.75) in children with IgM negative results. This finding was statistically significant $(P$ value $=0.018)$.

Totally, 60 patients $(24 \%)$ had positive serology tests (IgM and/or IgG) in our survey. Among them, 35 cases had negative SARS-CoV-2 RT-PCR result (58\%) and 24 $(40 \%)$ had underlying disease $(P$ value $=0.07)$. Out of 60 seropositive patients, $14(23 \%)$ experienced severe disease $(P$ value $=0.5)$. The ventilator was utilized in $6(10 \%)$ of such patients $(P$ value $=1.0)$. Intubation was carried out in $3(5 \%)$ of patients $(P$ value $=$ $0.769)$. ICU admission occurred in $11(18.3 \%)$ of our seropositive patients $(P$ value $=0.59)$. Mortality happened in $4(6.7 \%)$ of these patients $(P$ value $=1)$. No significant relationship was discovered between SARSCoV-2 seropositivity and clinical manifestations of the disease (Table 2). Table 3 compares laboratory tests in seropositive and seronegative patients. Lower level of 
platelets in IgG positive in comparison with IgG negative group ( $P$ value: 0.015$)$. Other than that, higher Alanine Aminotransferase (ALT) was observed more in the mentioned group ( $P$ value: 0.02$)$; however, mostly the ALT values were considered in the normal range. In patients with the positive $\operatorname{IgM}$, relative hypocalcemia (median of 8.25; IQR: 8.02-8.62) was found which appeared to be significant ( $P$ value: 0.02 ).

\subsection{Discussion}

COVID-19 is either infrequent in children or it has not been diagnosed that often, as this age group remains asymptomatic. Although the pathogenesis of COVID-19 is not fully known [15-17], a significantly smaller number of reported cases of COVID-19 in the pediatric population compared with the adults was reported $[18,19]$. The possible mechanisms might be due to the differences in Angiotensin -converting enzyme 2 (ACE2) expression, innate immunity, and the influence of preventive strategies including closure of schools and day-care centers [20]. However, increased availability of PCR tests even for milder cases has played a great role in patient detection [1]. Li et al. suggested that $86 \%$ of all early COVID-19 infections in China remained undiagnosed, while undocumented cases may have a lower transmission rate, their extensive number indicates that they could have been the origin of $79 \%$ of all early cases. Accordingly, children with few symptoms could be hazardous for their elderly caregivers having a predisposing risk factor for COVID-19 [21,22].

Our research is similar to previous studies depicting that fever and cough are the leading clinical manifestations of COVID-19 in children [14,19,23]. Nonetheless, tachypnea and respiratory distress were the other prevalent symptoms detected in children's age group. This finding is also favorable to a systematic review and meta-analysis in the pediatric field [23]. Nearly half of our patients suffered from an underlying disease including cardiovascular or immunodeficiency disorders and malignancies which was consistent with other reports [24].

In our study, only $35 \%$ of highly suspected patients for COVID-19 had a positive RT-PCR result. Other evidence strongly supports this data and raises concern about frequent false negative RT-PCR results [14,25]. The total positive rate of $30-60 \%$ for throat swab RTPCR has been reported at initial presentation of patients with COVID-19 infection [26]. Similar to previous reports [14,27], most children presented with MIS-C display no detectable viral infection by RT-PCR.
Weisberg and colleagues have proposed a differentiated infection course and immune response in children independent of whether they develop MIS-C, with implications for establishment of age-targeted strategies used in testing and protecting the population [10]. Our study advocates the lower titers of seropositivity in children as well.

The median time between fever onset and IgM seropositivity was significantly relevant in our survey, with a median of 7 days between fever onset and IgM seropositivity detected, which was consistent with other reports in adults. These findings emphasize that IgM antibodies can be used to indicate current or recent infections [28].

In our study, $35 \%$ of patients were confirmed by SARS CoV-2 RT-PCR and 24\% had positive serology tests (IgM and/or IgG). In previous report from a tertiary children's hospital in India, 13\% $(n=62)$ had positive SARS CoV-2 RT-PCR and 91 out of 463 children $(19.6 \%)$ were seropositive [29]. In our study, only $12 \%$ of the patients presented with MIS-C had a detectable IgG that was lower than previous reports [29]. There were no differences in antibody profiles between children with and without MIS-C; thus, the adaptive immune response may not be associated with MIS-C pathogenesis [10].

The reduced functional antibody response in children compared to adults could also be due to the efficacious immune-mediated viral clearance resulting in fewer respiratory symptoms and severe illness [10].

In this study, positive SARS-CoV-2 IgG was significantly related to thrombocytopenia and elevated ALT. Three mechanisms of thrombocytopenia have been hypothesized beforehand. First, direct infection of bone marrow cells by the virus and inhibition of platelet synthesis, second, reduction of platelet synthesis due to lung injury and third, platelet destruction by the immune system. The third mechanism might lead to elevated antibodies of IgG and thrombocytopenia. Antibodies and immune complexes deposited on the surfaces of platelets will be recognized by reticuloendothelial cells, and the platelets will be destroyed as target tissues, resulting in excessive platelet destruction [30].

As described by preliminary reports, patients presented with hypocalcemia were more severely ill on admission and had worse outcomes [31]. Positive SARSCoV-2 IgM and hypocalcemia indicated a significant relationship in our investigation. 


\section{Conclusion}

This is the first study describing the SARS-CoV-2 seropositivity among children in Iran and provides important insight about the COVID-19 infection in children. Our study advocates the lower titers of seropositivity in children in comparison to adults. Thrombocytopenia and high ALT could be compatible with positive SARS-CoV-2 IgG. Moreover, positive SARS-CoV-2 $\mathrm{IgM}$ and hypocalcemia indicated a significant relationship in our investigation.

\section{Limitations}

As this research was executed among admitted and highly suggestive for COVID-19 children, clinical manifestations and paraclinical outcomes may not be extensible to all patients infected with the disease. Performing bigger multi-centric studies including both outpatient and inpatient children with COVID-19 diagnosis could add more specifics to the literature.

\section{Conflict of interest}

The authors have declared no competing interest.

\section{Acknowledgments}

This study was supported by a grant (grant number: 99-1-149-47192) from Tehran University of Medical Sciences to Dr. Setareh Mamishi.

\section{References}

[1] G. Gharehbaghi, S. Yousefzadegan, A. Javid, H. RiaziEsfahani, A. Mousavi, S. Mahdavynia, R. Zare Mahmoudabadi, M. Radgoodarzi and M. Vafaee-Shahi, Iranian Journal of Pediatrics 31 (2021).

[2] A.M. Sola, A.P. David, K.W. Rosbe, A. Baba, L. RamirezAvila and D.K. Chan, JAMA Pediatrics 175 (2021), 198.

[3] N.S. Mehta, O.T. Mytton, E.W. Mullins, T.A. Fowler, C.L. Falconer, O.B. Murphy, C. Langenberg, W.J. Jayatunga, D.H Eddy and J.S. Nguyen-Van-Tam, Clinical Infectious Diseases 71 (2020), 2469.

[4] H. Tezer and T.B. Demirdağ, Turkish Journal of Medical Sciences 50 (2020), 592

[5] P.C. Williams, A.R. Howard-Jones, P. Hsu, P. Palasanthiran, P.E. Gray, B.J. McMullan, P.N. Britton and A.W. Bartlett, Pathology (2020).
[6] T. Alsaied, A.H. Tremoulet, J.C. Burns, A. Saidi, A. Dionne, S.M. Lang, J.W. Newburger, S. de Ferranti and K.G. Friedman, Circulation 143 (2021), 78.

[7] S.P. Adhikari, S. Meng, Y.-J. Wu, Y.-P. Mao, R.-X. Ye, Q.-Z. Wang, C. Sun, S. Sylvia, S. Rozelle and H. Raat, Infectious Diseases of Poverty 9 (2020), 1.

[8] C.S. Guan, Z.B. Lv, S. Yan, Y.N. Du, H. Chen, L.G. Wei, R.M. Xie and B.D. Chen, Academic Radiology 27 (2020), 609.

[9] M. Rezaei, M. Sadeghi, A. Korourian, P. Tabarsi, M. Porabdollah, E. Askari, E. Mortaz, S. Mahmoudi and M. Marjani, Human Antibodies (2021), 1.

[10] S.P. Weisberg, T.J. Connors, Y. Zhu, M.R. Baldwin, W.-H. Lin S. Wontakal, P.A. Szabo, S.B. Wells, P. Dogra and J. Gray, Nature Immunology 22 (2021), 25.

[11] T. Waterfield, C. Watson, R. Moore, K. Ferris, C. Tonry, A. Watt, C. McGinn, S. Foster, J. Evans and M.D. Lyttle, Archives of Disease in Childhood (2020).

[12] M. Cattalini, A. Taddio, C. Bracaglia, R. Cimaz, S. Della Paolera, G. Filocamo, F. La Torre, B. Lattanzi, A. Marchesi and G. Simonini, Italian Journal of Pediatrics 47 (2021), 1.

[13] M. Ekbatani, S. Hassani, L. Tahernia, B. Yaghmaei, S. Mahmoudi, A. Navaeian, M. Rostamyan, F. Zamani and S. Mamishi, British Journal of Biomedical Science 78 (2021), 47.

[14] S. Mamishi, Z. Movahedi, M. Mohammadi, V. Ziaee, M. Khodabandeh, M.R. Abdolsalehi, A. Navaeian, H. Heydari, S. Mahmoudi and B. Pourakbari, Epidemiology \& Infection 148 (2020).

[15] S. Mahmoudi, B. Yaghmaei, M. Sharifzadeh Ekbatani, B. Pourakbari, A. Navaeian, N. Parvaneh, M.T. Haghi Ashtiani and S. Mamishi, Frontiers in Pediatrics 9 (2021), 267.

[16] M. Rezaei, M. Marjani, S. Mahmoudi, E. Mortaz and D. Mansouri, International Archives of Allergy and Immunology 182 (2021), 254

[17] S. Mahmoudi, M. Rezaei, N. Mansouri, M. Marjani and D. Mansouri, Journal of Clinical Immunology 40 (2020), 974.

[18] E.H.E. Memar, S. Mamishi, M.S. Ekbatani, H. Alimadadi, B. Yaghmaei, V. Chegini, S. Janani and S. Mahmoudi, Archives de Pdiatrie 27 (2020), 502.

[19] S. Mamishi, H. Heydari, A. Aziz-Ahari, M.R. Shokrollahi, B. Pourakbari, S. Mahmoudi and Z. Movahedi, Journal of Microbiology, Immunology and Infection (2020).

[20] N. Dhochak, T. Singhal, S. Kabra and R. Lodha, The Indian Journal of Pediatrics 87 (2020), 537.

[21] J.F. Ludvigsson, Acta Paediatrica 109 (2020), 1088.

[22] S. Mahmoudi, M. Rostamyan, A. Aziz-Ahari, B. Pourakbari, M. Ghaffari and S. Mamishi, Tanaffos 19 (2020), 156.

[23] A. Gholami, R. Hemati, A. Khorshidi, M. Borji, M. Kafashian, G. Kalvandi, A. Tarjoman, S. Mahdikhani and M. Shokri, International Journal of Pediatrics 8 (2020), 12177.

[24] D. Sun, H. Li, X.-X. Lu, H. Xiao, J. Ren, F.-R. Zhang and Z.-S. Liu, World Journal of Pediatrics (2020), 1.

[25] S. Woloshin, N. Patel and A.S. Kesselheim, New England Journal of Medicine 383 (2020), e38.

[26] Y. Yang, M. Yang, C. Shen, F. Wang, J. Yuan, J. Li, M. Zhang, Z. Wang, L. Xing and J. Wei, MedRxiv (2020).

[27] V. Sancho-Shimizu, P. Brodin, A. Cobat, C.M. Biggs, J. Toubiana, C.L. Lucas, S.E. Henrickson, A. Belot and S.G. Tangye, Journal of Experimental Medicine 218 (2021), e20210446.

[28] M. Yan, Y. Zheng, Y. Sun, L. Wang, L. Luan, J. Liu, X. Tian and N. Wan, Journal of Medical Virology 93 (2021), 441.

[29] A. Venkataraman, S. Balasubramanian, S. Putilibai, S. Lakshan Raj, S. Amperayani, S. Senthilnathan, A. Manoharan, 
A. Sophi, R. Amutha and K. Sadasivam, Journal of Tropical Pediatrics 67 (2021), fmab015.

[30] P. Xu, Q. Zhou and J. Xu, Annals of Hematology 99 (2020), 1205.
[31] B. Torres, P. Alcubilla, A. Gonzlez-Cordn, A. Inciarte, M. Chumbita, C. Cardozo, F. Meira, M. Gimnez, A. de Hollanda and A. Soriano, International Journal of Infectious Diseases 104 (2021), 164. 